\title{
Does COVID-19 Policy Affect Initiation and Duration of Exclusive Breastfeeding? A Single-center Retrospective Study
}

\author{
Abeer Salem Al Shahrani (D) \\ Department of Clinical Sciences, College \\ of Medicine, Princess Nourah Bint \\ Abdulrahman University, Riyadh, Saudi \\ Arabia
}

Purpose: It assessed the effect of a COVID-19 policy that involved maternal-neonatal separation on early initiation and duration of exclusive breastfeeding (EBF) at two weeks, three, and six months postpartum during the peak of the COVID-19 pandemic in 2020.

Patients and Methods: This retrospective study included consecutive mother-newborn dyads with deliveries between mid-March and the end of December 2020 who agreed to participate and were eligible according to the following criteria: full-term pregnancy, singleton birth, and healthy newborn. Participants were called at least twice during the study period to inquire about exclusive breastfeeding status at two weeks, three, and six months postpartum. Multivariate logistic regression analysis was carried out to identify associated factors with EBF.

Results: A total of 454 eligible mother-infant dyads were included. Mean maternal age (in years), gestational age (in weeks), birth weight (in kilograms), and 5-min Apgar score was, respectively, $32 \pm 5.4$ years, $39 \pm 1.3$ weeks, $3.1 \pm 0.42 \mathrm{~kg}$, and $9.24 \pm 0.6$. Most of the mothers (86.1\%) had no known chronic diseases and had a normal spontaneous vaginal delivery (NSVD, 91.4\%). Approximately $44.7 \%$ of the sample initiated breastfeeding either directly or with expressed breastmilk during their hospital stay. Prevalence of EBF during the first two weeks, three, and six months postpartum was, respectively, $30 \%, 19.8 \%$, and $31.7 \%$. EBF was associated with birth weight, hospital breastfeeding and EIBF at early postpartum period, while EBF at six months was associated with parity and method of delivery.

Conclusion: During the peak of the COVID-19 pandemic, the prevalence of breastfeeding initiation and EBF in the first six months postpartum were low among Saudi mothers. However, multicenter, prospective, cohort studies with adjustment for known confounding factors are required to explore the impact of infection control policies on breastfeeding. Meanwhile, these policies should support early and safe breastfeeding practices, especially with new and evolving information regarding the current pandemic.

Keywords: COVID-19, pandemic, breastfeeding, exclusive breastfeeding, policy

\section{Plain Language Summary}

COVID-19 pandemic has placed a major burden on health-care facilities and significantly disrupted societies and economies worldwide. Early in the pandemic, there was uncertainty about SARS-CoV-2 transmission, including vertical transmission. Thus, multiple health-care facilities around the world adopted strict infection control policies that involved motherneonate separation in case of suspected or positive COVID-19 PCR. After one year of this pandemic, with the lack of evidence on viral transmission through breastmilk, multiple guidelines encourage breastfeeding initiation and rooming-in, regardless of the maternal
Correspondence: Abeer Salem Al Shahran Department of Clinical Sciences, College of Medicine, Princess Nourah bint Abdulrahman University, PO Box 84428, Riyadh II67I, Saudi Arabia,

Tel +9660I I823903I

Email asalshahran@pnu.edu.sa 
COVID-19 status. Despite the availability of updated guidelines, multiple institutes worldwide still implement strict infection control measures, which involve temporary mother-neonate separation. Furthermore, consequences of such measures on early breastfeeding initiation and EBF duration have not been fully explored. In our study, we found that the prevalence of breastfeeding initiation and EBF in the first six months postpartum was low among Saudi mothers in a single center in Riyadh. This study will help health-care policy makers as well as health-care providers better understand the consequences of infection control policies on breastfeeding and, hence, improve the preparedness for the current pandemic, especially with the newly emerging SARS-CoV-2 strains.

\section{Introduction}

Breastfeeding has short and long-term benefits both for mother and infant. ${ }^{1,2}$ However, these benefits are dose dependent and increase with the exclusivity and a longer duration of breastfeeding. ${ }^{3}$ The best way to promote successful breastfeeding from day one, is to ensure that the mother-newborn dyad is kept together, and skin-to-skin contact is supported. ${ }^{4}$ Thus, the World Health Organization (WHO) recommends that breastfeeding is initiated within an hour of birth, exclusive breastfeeding (EBF) is performed for the first six months, and breastfeeding is continued for at least two years. ${ }^{5}$

Nowadays, the world is still suffering from the consequences of the coronavirus disease 2019 (COVID-19), in which several restrictions were implemented to contain the spread of the pandemic. Indeed, breastfeeding was no exception. Early in the pandemic, there were concerns about transmitting the virus to newborns and infants through breastfeeding. ${ }^{6}$ Thus, health-care facilities around the world implemented strict infection control measures to prevent disease transmission, including mother-newborn separation if COVID-19 was suspected or confirmed, as well as limited visitation and parental presence in labor and neonatal wards. ${ }^{7}$ In addition, there has been a decline in the number of supportive and group breastfeeding activities. ${ }^{8}$

In the absence of clear evidence of vertical transmission of SARS-CoV-2, ${ }^{9}$ current recommendations of the WHO, UNICEF, United Nations Population Fund (UNFPA) and Academy of Breastfeeding Medicine (ABM) state that mothers and infants should remain together (rooming-in) and engage in continuous skin-to-skin contact, particularly immediately after birth during the initiation of breastfeeding, despite suspected, probable, or confirmed COVID-19. ${ }^{10}$ Moreover, they reinforce the importance of providing expressed breastmilk for infants of severely ill mothers. ${ }^{11}$ However, these recommendations have not been strictly followed by health-care facilities due to variations in infection rates, the availability of health-care resources, and continuously evolving scientific data. ${ }^{12}$

Labor and delivery units are critical wards in all healthcare institutes where the risk of transmission of COVID-19 is high due to the unplanned nature of labor and arrival of mothers who are not tested, yet they could be exposed or infected. While the majority of guidelines implementing precautions such as wearing personal protective equipment (PPE), social distancing and screening the women who are admitted for labor. In contrast, a limited number of associations/scientific groups implemented temporary mothernewborn separation as an infection control measure, such as US CDC, ${ }^{13}$ and Chinese Pediatric COVID-19 Working Group. ${ }^{14}$ Moreover, organizations and societies agree about the temporary separation when the COVID-19 positive mother is too sick to care for her baby. ${ }^{15}$ Although, separation may minimize the risk of COVID-19 transmission during the hospital stay, however, it will negatively impact the physical and mental health of mothers and newborns. ${ }^{16,17} \mathrm{~A}$ recent report has found that maternal-infant separation, whether the mother was COVID-19 positive or not, was associated with negative breastfeeding experiences. ${ }^{18}$

In Saudi Arabia, multiple observational studies reported poor breastfeeding practices, such as delayed breastfeeding initiation, ${ }^{19}$ relatively high prevalence of mixed feeding, ${ }^{20}$ and decline in duration/lacking exclusivity of breastfeeding ${ }^{21,22}$ compared to old literature. ${ }^{23}$ To the best of our knowledge, no published local study assessed breastfeeding practices during the pandemic. Furthermore, it is unclear whether infection control policies that took place during the peak of the COVID-19 pandemic will negatively affect breastfeeding during the first six months postpartum. Therefore, we aimed to assess the effect of the local COVID-19 policy on breastfeeding initiation and duration at two weeks, three, and six months postpartum during the peak of the COVID-19 pandemic and just before the start of the COVID-19 national vaccination campaign.

\section{Patients and Methods}

\section{Study Design, Setting and Participants}

This was a single-center, retrospective observational study that was conducted in Riyadh, Saudi Arabia, between August 2020 and August 2021. The study was carried 
out at a university hospital in which the COVID-19 policy was effectively implemented in mid-March 2020. The policy took into consideration multiple aspects of maternal and neonatal care at different stages and included the requirement for a COVID-19 PCR test for all admitted patients despite active labor or elective admission. Patients with suspected or confirmed COVID-19 were admitted to dedicated wards with dedicated labor and delivery rooms, and a similar procedure was applied for neonates of mothers with suspected/confirmed COVID-19. Concerning skin-skin contact and breastfeeding, no skinskin contact was allowed, and direct breastfeeding was not advised if the mother's COVID-19 status was suspected or confirmed. However, extracting breastmilk was allowed with precautionary measures and the help of health-care providers to feed newborns.

The study included all mother-newborn dyads who agreed to participate and were eligible according to the following criteria: full-term pregnancy (ie, gestational age from 37 to 42 completed weeks), singleton birth, and healthy newborn as defined by normal birth weight $(\geq 2.5$ to $<4.0 \mathrm{~kg}),{ }^{24}$ and a five-minute Apgar score of 8 or higher.

\section{Data Collection and Study Variables}

We have enrolled consecutively all births that took place from 15 March 2020 until the end of December 2020. However, data collection and follow-up calls were continued until the end of August 2021. Using preliminary data in the labor unit registry, participants were screened for eligibility, and if they were eligible, their electronic medical records were further reviewed for validation of information by a trained unit assistant. In addition, all participants' contact information was recorded to allow follow-up telephone calls at three and six months postpartum. They were called at least twice during the study period, and if they had been called more than three times with no answer, they were considered lost to follow-up.

The following data were collected using an anonymous tool generated by RedCap ${ }^{\circledR}$ : maternal age, gestational age, parity, chronic diseases, method of delivery, birth weight in kilograms, five-minute Apgar score, maternal COVID19 status, length of hospital stay, breastfeeding during hospital stay, and breastfeeding initiation time. Furthermore, participants were interviewed via telephone call by trained staff to verify the abovementioned information and obtain information about exclusive breastfeeding (EBF) status at two weeks, three, and six months postpartum using Yes or No questions. EBF was defined as: direct or expressed breastmilk provided, with or without oral rehydration salts (ORS) or drops or syrups containing vitamins, minerals, or medicines. ${ }^{25}$ Early initiation of breastfeeding (EIBF) was defined as initiation of breastfeeding within one hour after delivery, based on the maternal report, as well as labor/delivery unit records, for those who were initiated EBF after one hour we further categorize the time into three intervals: $2-23,24-48$ hours, and $>48$ hours after delivery.

\section{Statistical Analysis}

SPSS version 21.0 (released 2012. IBM SPSS Statistics for Windows, IBM Corporation, Armonk, NY, USA) was used for statistical analysis. Descriptive statistics (frequencies, percentages, means, and standard deviations) were calculated to describe categorical and numerical variables. EBF rate was calculated as per WHO formula. ${ }^{26}$ Chi-squared/ Fisher's exact tests were used as appropriate, to quantify difference between the two groups (EBF, No EBF) at two weeks, three, and six months postpartum. Furthermore, logistic regression was done for all independent variables, to identify associated factors with EBF. The associations were reported by the odds ratio (OR) with $p$-values and $95 \%$ confidence interval $(95 \% \mathrm{CI})$ were used to report the statistical significance and precision of the results, respectively. The model was checked for the goodness-of-fit (GOF) using Hosmer-Lemeshow test; $p$-values $\leq 0.05$ were considered statistically significant.

\section{Results}

\section{Sociodemographic Characteristics of Study Population}

A total of 454 eligible mother-infant dyads were included, however, 273 completed the study until its end. Mean maternal age (in years), gestational age (in weeks), birth weight (in kilograms), and five-minute Apgar score was, respectively, $32 \pm 5.4$ years, $39 \pm 1.3$ weeks, $3.1 \pm 0.42 \mathrm{~kg}$, and $9.24 \pm 0.6$. The majority of mothers $(86.1 \%)$ had no known chronic diseases and had a normal spontaneous vaginal delivery (NSVD, 91.4\%). Moreover, 97.2\% of mothers had negative COVID-19 PCR result on admission. Approximately $44.7 \%$ of the sample breastfed their newborns either directly or through expressed breastmilk during their stay in the hospital; in which, $24.2 \%$ initiated breastfeeding within one hour (Table 1). 
Table I Sociodemographic and Clinical Characteristics of the Study Population $(n=454)$

\begin{tabular}{|c|c|}
\hline Variable & Distribution n (\%) \\
\hline \multicolumn{2}{|c|}{ Maternal age (years) } \\
\hline $18-24$ & $61(13.4)$ \\
\hline $25-34$ & $299(65.9)$ \\
\hline $35-45$ & $94(20.7)$ \\
\hline \multicolumn{2}{|c|}{ Parity } \\
\hline 0 & $105(24.3)$ \\
\hline $\mathrm{I}-2$ & $285(66.0)$ \\
\hline 3 or more & $42(9.7)$ \\
\hline \multicolumn{2}{|c|}{ Chronic diseases } \\
\hline No & $382(84.1)$ \\
\hline Yes & $72(15.9)$ \\
\hline \multicolumn{2}{|c|}{ Gestational age (weeks) } \\
\hline $37-39$ & $265(58.4)$ \\
\hline $40-42$ & $189(4 \mid .6)$ \\
\hline \multicolumn{2}{|c|}{ Method of delivery } \\
\hline NSVD & $4 \mid 3(9 \mid .0)$ \\
\hline Vacuum & $12(2.6)$ \\
\hline $\mathrm{C} / \mathrm{S}$ & $29(6.4)$ \\
\hline \multicolumn{2}{|c|}{ Birth weight (kg) } \\
\hline $2.50-2.99$ & $123(27.1)$ \\
\hline $3.00-3.49$ & $199(43.8)$ \\
\hline $3.50-4.00$ & $132(29.1)$ \\
\hline \multicolumn{2}{|c|}{ Hospital breastfeeding } \\
\hline No & $25 I(55.3)$ \\
\hline Yes & $203(44.7)$ \\
\hline \multicolumn{2}{|c|}{ Early Initiation of breastfeeding (EIBF) } \\
\hline No & $344(75.8)$ \\
\hline Yes & $110(24.2)$ \\
\hline \multicolumn{2}{|c|}{ Initial breastfeeding time (hospital) } \\
\hline $2-23$ hours & $45(9.9)$ \\
\hline 24-48 hours & $29(6.4)$ \\
\hline$>48$ hours & $16(3.5)$ \\
\hline
\end{tabular}

Based on the labor/delivery ward census, skin-skin and EIBF varied according to the total number of deliveries, which declined due to the implementation of strict eligibility criteria for hospital admission as well as the implementation of the COVID-19 policy (Figure 1).

Regarding prevalence of exclusive breastfeeding (EBF) among the study population, it was found that $30 \%$ of mothers breastfeed their newborns in the first two weeks postpartum, and the percentage decreased to $19.8 \%$ in the first three months postpartum, as shown in Figure 2. However, the prevalence of EBF increased after six months postpartum (31.7\%). Moreover, approximately half of mothers who tested positive for COVID-19, continued exclusive breastfeeding up to six months postpartum.

Table 2 describes the characteristics of the subgroup who continued the study $(\mathrm{n}=273)$. There was a statistically significant difference between mothers who exclusively breastfed their infants and those who did not, this was observed with the following factors: maternal age, method of delivery, hospital breastfeeding, and EIBF. There was no statistically significant difference between the abovementioned groups across other characteristics: parity, chronic diseases, birth weight, maternal COVID-19 test, and length of hospital stay.

Furthermore, we have carried out univariate logistic regression to determine potential associated factors with exclusive breastfeeding. It was found that the EBF differed across demographic and clinical characteristics, in which EBF was linked to parity, gestational age, method of delivery, birth weight, hospital BF and EIBF (Table 3). Moreover, multivariate logistic regression analysis for all these factors had attenuated the effect of parity, birth weight, methods of delivery, hospital breastfeeding and EIBF (Table 4). However, important factors such as maternal age and maternal COVID-19 status were not significantly associated with EBF.

\section{Discussion}

During the first year of the COVID-19 pandemic, varying levels of support for breastfeeding and the motherinfant dyad were reflected in policies and guidelines. ${ }^{7}$ While some precautions were proven to be of value, others such as temporary mother-newborn separation, were having negative impact both on mother and newborns. ${ }^{27}$ Only limited studies, including this study, have explored the consequences of temporarily mothernewborn separation on breastfeeding. ${ }^{28,29}$ Our study shed light on the effect of the COVID-19 policy on EBF initiation and duration during the first six months postpartum. We observed variation in the EBF prevalence at different times during the postpartum period. However, it is considered low compared to international figures, ${ }^{30}$ with $19.8 \%$ of the sample was exclusively 


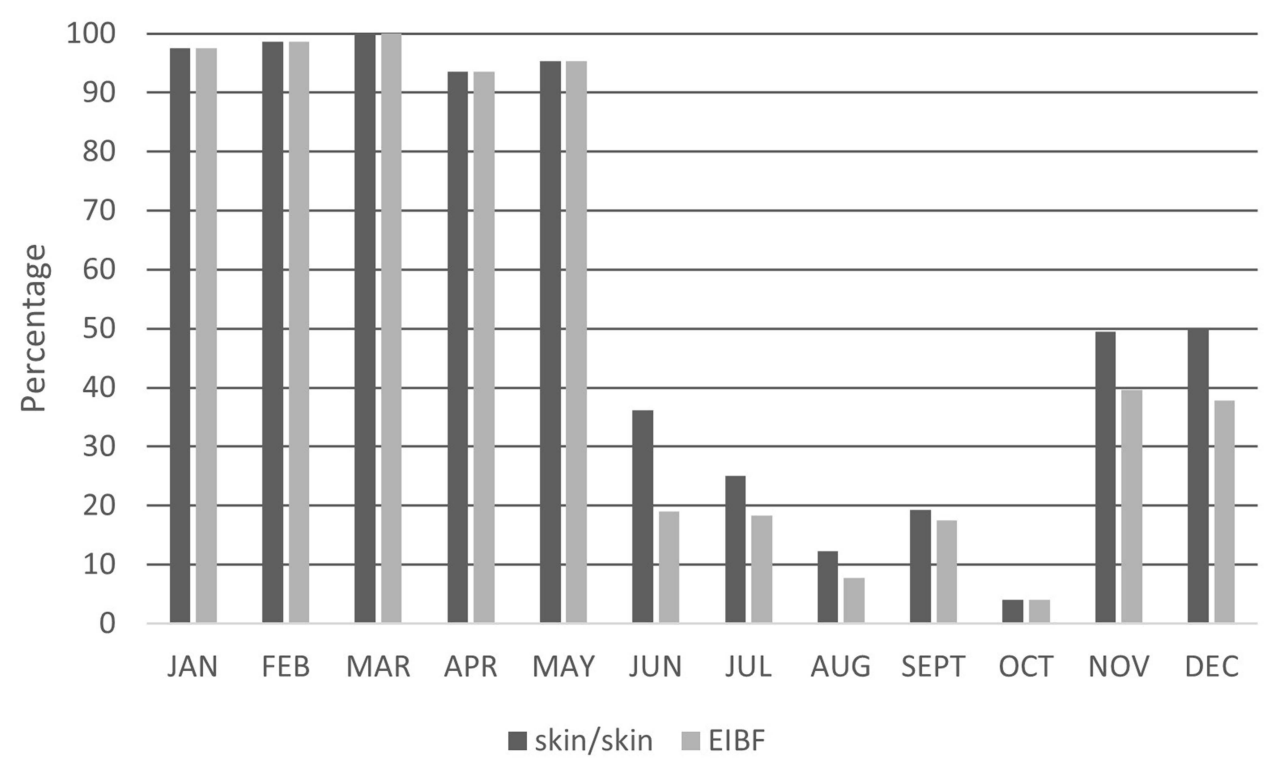

Figure I Prevalence of skin-skin and EIBF in KAAUH, from January to December 2020.

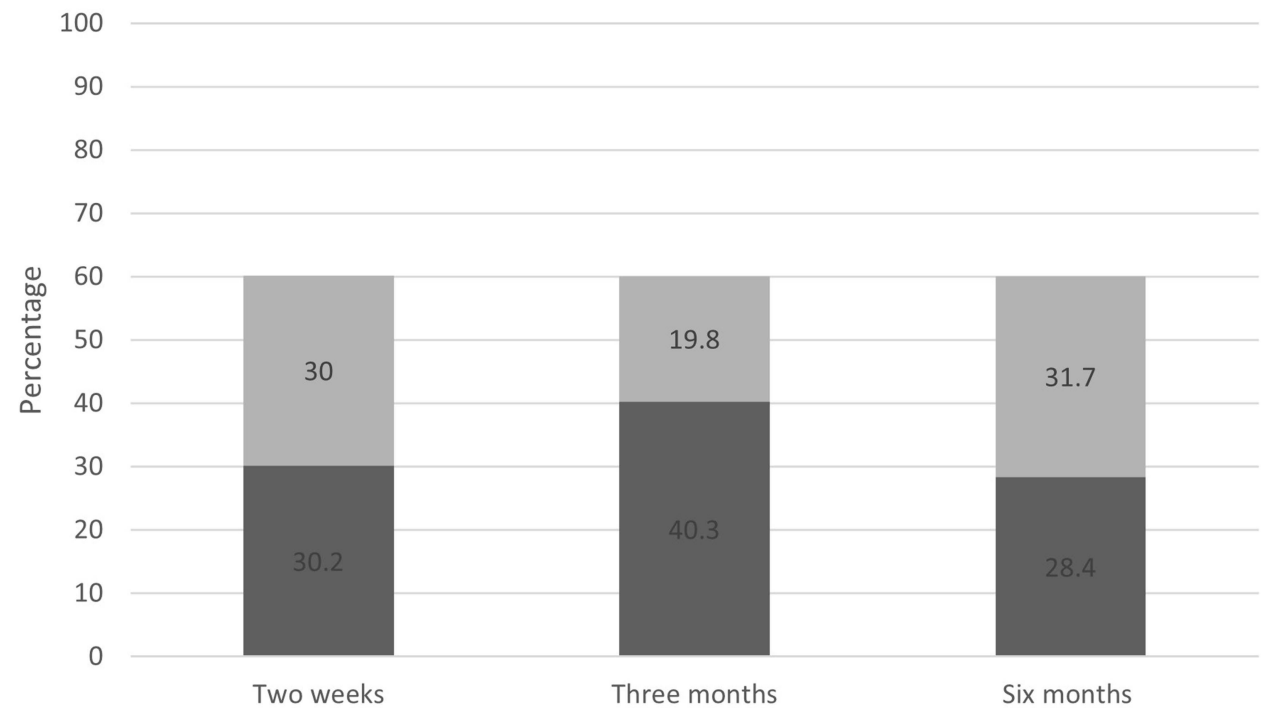

Exclusive Breastfeeding

No $=$ Yes

Figure 2 Exclusive breastfeeding status at two weeks, three, and six months postpartum among the study population.

breastfeeding as early as three months postpartum. Interestingly, there was a marked improvement in the EBF status at six months postpartum (31.7\%). This observation was consistent with another cohort study done in a tertiary center in India. ${ }^{31}$ However, to justify this finding we need to explore other factors that might have contributed to EBF duration.
Although the decision to breastfeed is highly personal, the initiation and duration of breastfeeding are often influenced by multiple factors. ${ }^{32}$ Nevertheless, events that occur during the first few days and weeks postpartum may promote or discourage breastfeeding. ${ }^{33,34}$ In some settings, COVID19 policies that implemented separation, have resulted in delayed initiation and disruption of breastfeeding. ${ }^{35}$ The 
Table 2 Sociodemographic and Clinical Characteristics with Exclusive Breastfeeding (EBF) at Two Weeks, Three, and Six Months Postpartum $(n=273)$

\begin{tabular}{|c|c|c|c|c|c|c|c|c|c|}
\hline \multirow[t]{3}{*}{ Factors } & \multicolumn{2}{|c|}{ EBF at 2 Weeks } & \multirow[t]{3}{*}{$p$-value } & \multicolumn{2}{|c|}{ EBF at 3 Months } & \multirow[t]{3}{*}{$p$-value } & \multicolumn{2}{|c|}{ EBF at 6 Months } & \multirow[t]{3}{*}{$p$-value } \\
\hline & No & Yes & & No & Yes & & No & Yes & \\
\hline & n (\%) & n (\%) & & n (\%) & n (\%) & & n (\%) & n (\%) & \\
\hline \multicolumn{10}{|c|}{ Maternal age (years) } \\
\hline $18-24$ & $20(17.1)$ & $13(1 \mid .5)$ & 0.48 & $31(20.4)$ & $2(2.6)$ & $0.001 *$ & $16(14.8)$ & 17 (13.9) & 0.1 \\
\hline $25-34$ & 91 (77.8) & $94(83.2)$ & & $112(73.6)$ & $73(93.6)$ & & $90(83.3)$ & 95 (77.9) & \\
\hline $35-45$ & $6(5.1)$ & $6(5.3)$ & & $9(6.0)$ & $3(3.8)$ & & $2(1.85)$ & $10(8.2)$ & \\
\hline \multicolumn{10}{|c|}{ Parity } \\
\hline 0 & $41(32.0)$ & $27(20.4)$ & 0.09 & $53(30.6)$ & $15(19.2)$ & 0.06 & $36(28.6)$ & $32(23.9)$ & 0.25 \\
\hline $\mathrm{I}-2$ & $77(60.2)$ & $91(69.0)$ & & $106(61.3)$ & $62(71.3)$ & & $82(65.1)$ & $86(64.2)$ & \\
\hline 3 or more & $10(7.8)$ & $14(10.6)$ & & $14(8.1)$ & $10(11.5)$ & & $8(6.3)$ & $16(11.9)$ & \\
\hline \multicolumn{10}{|c|}{ Chronic diseases } \\
\hline No & $123(89.8)$ & $123(90.4)$ & 0.85 & $165(90.2)$ & $81(90.0)$ & 0.97 & II4 (88.4) & 132 (9|.7) & 0.36 \\
\hline Yes & $14(10.2)$ & $13(9.6)$ & & $18(9.8)$ & $9(10.0)$ & & $15(11.6)$ & $12(8.3)$ & \\
\hline \multicolumn{10}{|c|}{ Method of delivery } \\
\hline NSVD & $128(93.4)$ & $117(86.0)$ & $0.04 *$ & 168 (91.8) & $77(85.6)$ & 0.11 & $110(85.3)$ & 135 (93.7) & $0.03^{*}$ \\
\hline None SVD & $9(6.6)$ & $19(14)$ & & $15(8.2)$ & $13(14.4)$ & & $19(14.7)$ & $9(6.3)$ & \\
\hline \multicolumn{10}{|c|}{ Birth weight (kg) } \\
\hline $2.50-2.99$ & $39(31.7)$ & $36(30.0)$ & 0.76 & $57(35.4)$ & $18(21.9)$ & 0.06 & $40(36.0)$ & $35(26.5)$ & 0.27 \\
\hline $3.00-3.49$ & $61(49.6)$ & $57(47.5)$ & & $76(47.2)$ & $42(51.2)$ & & $50(45.0)$ & $68(51.5)$ & \\
\hline $3.50-4.00$ & $23(18.7)$ & $27(22.5)$ & & $28(17.4)$ & $22(26.8)$ & & $21(19.0)$ & $29(22.0)$ & \\
\hline \multicolumn{10}{|c|}{ Hospital breastfeeding } \\
\hline No & $28(65.1)$ & $28(40.6)$ & 0.65 & $45(71.4)$ & $25(5 \mathrm{I})$ & $0.03 *$ & $59(67.8)$ & II (34.8) & $0.03 *$ \\
\hline Yes & $15(34.9)$ & $15(2 \mid .7)$ & & $18(28.6)$ & $24(49)$ & & $28(32.2)$ & $14(65.2)$ & \\
\hline \multicolumn{10}{|c|}{ Early Initiation of breastfeeding (EIBF) } \\
\hline No & $87(63.5)$ & $101(74.3)$ & $0.04 *$ & $118(64.5)$ & $70(77.8)$ & $0.03 *$ & $98(76.0)$ & $90(62.5)$ & $0.02 *$ \\
\hline Yes & $50(36.5)$ & $35(25.7)$ & & $65(35.5)$ & $20(22.2)$ & & $31(24.0)$ & $54(37.5)$ & \\
\hline \multicolumn{10}{|c|}{ Maternal COVID-19 test } \\
\hline Negative & I 34 (97.8) & $133(97.8)$ & 0.9 & I $79(97.8)$ & $88(97.8)$ & 0.9 & $126(97.7)$ & |4| (98.0) & 0.8 \\
\hline Positive & $3(2.2)$ & $3(2.2)$ & & $4(2.2)$ & $2(2.2)$ & & $3(2.3)$ & $3(2.0)$ & \\
\hline \multicolumn{10}{|c|}{ Length of hospital stay } \\
\hline $0-24$ hours & $62(47.3)$ & $75(57.7)$ & 0.09 & $85(48.6)$ & $52(60.5)$ & 0.07 & $73(57.9)$ & $64(47.4)$ & 0.09 \\
\hline$>24$ hours & $69(52.7)$ & $55(42.3)$ & & $90(5 \mathrm{I} .4)$ & $34(39.5)$ & & $53(42.1)$ & 71 (52.6) & \\
\hline
\end{tabular}

Note: *p-value $<0.05$.

same applied for this study, these results highlight the need for further research into the association between COVID-19 policies, breastfeeding practices, and the long-term impacts of the pandemic on maternal and neonatal outcomes. Filling this knowledge gap will foster the development of guidelines and interventions to improve breastfeeding experience during the current COVID-19 pandemic.

There is insufficient evidence to support SARS-CoV-2 transmission through breastmilk, ${ }^{36}$ however, the current situation is challenging especially with newly emerging 
Table 3 Univariate Logistic Regression for Factors Associated with EBF Among the Study Population

\begin{tabular}{|c|c|c|c|c|c|c|}
\hline \multirow[t]{2}{*}{ Factors } & \multirow{2}{*}{$\frac{\text { EBF at } 2 \text { Weeks }}{\text { OR }(95 \% \mathrm{Cl})}$} & \multirow[t]{2}{*}{ p-value } & \multirow{2}{*}{$\begin{array}{c}\text { EBF at } 3 \text { Months } \\
\text { OR }(95 \% \mathrm{Cl})\end{array}$} & \multirow[t]{2}{*}{$p$-value } & \multirow{2}{*}{$\begin{array}{c}\text { EBF at } 6 \text { Months } \\
\text { OR }(95 \% \mathrm{Cl})\end{array}$} & \multirow[t]{2}{*}{$p$-value } \\
\hline & & & & & & \\
\hline \multicolumn{7}{|l|}{ All factors included } \\
\hline Maternal age & $1.03(0.98-1.08)$ & 0.17 & $1.03(0.98-1.08)$ & 0.18 & I.0I (0.97-I.06) & 0.6 \\
\hline Parity & $1.08(0.92-1.28)$ & 0.37 & I.I (0.92-I.30) & 0.3 & $1.23(1.04-1.46)$ & $0.01 *$ \\
\hline Chronic diseases (Ref. No) & $0.89(0.44-1.8)$ & 0.74 & $0.88(0.4|-| .88)$ & 0.74 & $\mathrm{I} .14(0.56-2.3 \mathrm{I})$ & 0.72 \\
\hline Gestational age & $1.02(0.85-1.23)$ & 0.81 & $1.11(0.91-1.35)$ & 0.28 & $1.23(1.02-1.48)$ & $0.03 *$ \\
\hline Method of delivery (Ref. Non-SVD) & $0.43(0.19-0.99)$ & $0.05^{*}$ & $0.53(0.24-1.16)$ & 0.11 & $0.39(0.17-0.89)$ & $0.025^{*}$ \\
\hline Birth weight & $1.08(0.59-1.98)$ & 0.8 & $2.18(1.13-4.19)$ & $0.02 *$ & $1.8(0.97-3.34)$ & 0.06 \\
\hline $5^{\prime}$ min Apgar score & $0.85(0.56-1.30)$ & 0.47 & $0.84(0.54-1.3 \mathrm{I})$ & 0.44 & $0.86(0.56-1.3 I)$ & 0.48 \\
\hline $\begin{array}{l}\text { Maternal COVID-19 test } \\
\text { (Ref. Negative) }\end{array}$ & I.0I $(0.20-5.08)$ & 0.99 & $1.02(0.18-5.67)$ & 0.98 & $0.89(0.18-4.5 \mathrm{I})$ & 0.9 \\
\hline Hospital length of stay & $0.93(0.79-1.1)$ & 0.39 & $0.89(0.74-1.08)$ & 0.25 & $1.18(0.98-1.4 I)$ & 0.07 \\
\hline Hospital breastfeeding (Ref. No) & $1.50(0.93-2.43)$ & 0.1 & $2.0(1.17-3.38)$ & $0.01 *$ & $1.08(0.67-1.74)$ & 0.76 \\
\hline EIBF (Ref. No) & $1.66(1.0-2.78)$ & $0.05^{*}$ & $1.93(1.1-3.45)$ & $0.03 *$ & $1.9(1.12-3.21)$ & $0.02 *$ \\
\hline
\end{tabular}

Note: $* p$-value $<0.05$.

Table 4 Multivariate Logistic Regression for Factors Associated with EBF Among the Study Population

\begin{tabular}{|c|c|c|c|c|c|c|}
\hline \multirow[t]{2}{*}{ Factors } & EBF at 2 Weeks & \multirow[t]{2}{*}{ p-value } & EBF at 3 Months & \multirow[t]{2}{*}{$p$-value } & EBF at 6 Months & \multirow[t]{2}{*}{$p$-value } \\
\hline & OR (95\%Cl) & & OR (95\%Cl) & & OR (95\%Cl) & \\
\hline \multicolumn{7}{|l|}{ All factors included } \\
\hline Maternal age & $1.02(0.96-1.08)$ & 0.46 & $1.02(0.95-1.08)$ & 0.62 & $0.98(0.93-1.04)$ & 0.61 \\
\hline Parity & $1.04(0.84-1.27)$ & 0.73 & $1.03(0.82-1.29)$ & 0.82 & $1.25(1.0-1.55)$ & $0.04 *$ \\
\hline Chronic diseases (Ref. No) & $0.86(0.4 \mathrm{I}-\mathrm{I} .84)$ & 0.65 & $0.9(0.38-2.12)$ & 0.82 & I.I4 (0.53-2.45) & 0.74 \\
\hline Gestational age & I.I (0.87-I.33) & 0.48 & $1.13(0.89-1.44)$ & 0.3 & I.I5 (0.93-I.42) & 0.21 \\
\hline Method of delivery (Ref. Non-SVD) & $2.0(0.8 I-4.96)$ & 0.14 & $1.60(0.63-4.10)$ & 0.32 & $0.39(0.16-0.99)$ & $0.05^{*}$ \\
\hline Birth weight & I.I (0.53-2.I) & 0.87 & $2.43(1.13-5.22)$ & $0.02 *$ & $\mathrm{I} .42(0.7 \mathrm{I}-2.84)$ & 0.32 \\
\hline Five-minute Apgar score & $0.92(0.58-1.47)$ & 0.71 & $0.87(0.52-1.46)$ & 0.6 & $0.78(0.47-1.22)$ & 0.3 \\
\hline Maternal COVID-19 test (Ref. Negative) & I.0I $(0.18-5.56)$ & 0.98 & $1.01(0.15-6.71)$ & 0.98 & $1.22(0.23-6.44)$ & 0.81 \\
\hline Hospital length of stay & $0.94(0.78-1.13)$ & 0.52 & $0.91(0.73-1.15)$ & 0.44 & $1.22(0.99-1.5 I)$ & 0.06 \\
\hline Hospital breastfeeding (Ref. No) & $2.93(1.53-5.5 \mathrm{I})$ & $0.001 *$ & $5.03(2.54-9.95)$ & $<0.00 I^{*}$ & $0.64(0.29-1.38)$ & 0.26 \\
\hline EIBF (Ref. No) & $2.90(1.39-6.01)$ & $0.004 *$ & $4.6(2.11-10.0)$ & $<0.001 *$ & $0.5 \mathrm{I}(0.25-1.06)$ & 0.07 \\
\hline
\end{tabular}

Note: $*$-value $<0.05$. 
strains of SARS-CoV-2. It has been reported that it has increased transmission rates compared to the original strain. Moreover, COVID-19 vaccines that were released by end of 2020, will require several months to become an effective control measure in the community. ${ }^{37}$

This study was carried out during the peak of the COVID-19 pandemic and ended just before the initiation of the COVID-19 national vaccination campaign. ${ }^{38}$ Thus, understanding COVID-19 epidemiology in Saudi Arabia might aid in the interpretation of our data, especially regarding the small number of confirmed COVID-19 cases among admitted mothers as well as the decline in skin-skin contact. According to an ecological study, the number of COVID-19 cases increased from one case on March 2 to 101,914 cases on June 7, 2020, representing an average of 1039 new cases per day; furthermore, new cases were more common among male adults. ${ }^{39}$ Another multicenter study carried out in Riyadh during the same period as that in our study showed that 288 pregnant mothers had confirmed COVID-19. ${ }^{40}$ Our study showed that $2.8 \%$ of mothers were positive for COVID-19; however, skin-skin contact decreased dramatically from $97 \%$ in May to $38 \%$ in June and continued to decline until the last quarter of 2020, when the WHO and UNICEF updated their guidelines for breastfeeding. ${ }^{11}$ Initially during the pandemic, skin-skin contact and rooming-in were allowed for mothers who were tested for COVID-19 within 24-48 hours of their arrival at the labor and delivery unit, whether from the emergency department or maternal ward; since the majority of our sample had a NSVD, carrying out a PCR test before mothers arrival was difficult, though it was possible in a few elective admissions. In addition, the estimated time to receive PCR test results ranged from 10-18 hours. Therefore, mothers with pending PCR results were admitted to the isolation ward until the test results were released.

Our study reported that EBF duration was significantly associated with both breastfeeding during hospital admission and early initiation of breastfeeding; however, only $44.7 \%$ of our sample were able to initiate breastfeeding during hospital admission, with $24 \%$ occurring within one hour of birth. The rest of the admitted mothers were unable to initiate breastfeeding in the hospital, either due to discharge within 24 hours (NSVD protocol) or delayed PCR test results. Furthermore, other associated factors with EBF are consistent with what is already known and firmly established in the literature such as birth weight, parity, and method of delivery. ${ }^{41,42}$
Finally, this study raises a concern about COVID-19 policies involving maternal-neonatal separation, which ultimately may affect early breastfeeding initiation and the optimal duration of EBF. However, we acknowledge that our study had several limitations, including its singlecenter, retrospective design, and $20-40 \%$ lost to follow-up rate. Although, we selected healthy, full-term, motherneonate dyads to eliminate effects of other maternal and neonatal comorbidities on breastfeeding initiation or duration. However, the effect of other confounding sociodemographic factors was not investigated, such as mother's knowledge and attitude regarding breastfeeding, maternal employment, and previous breastfeeding experience. These factors may influence or discourage breastfeeding, as reported in the literature. ${ }^{43,44}$ Thus, multicenter, prospective, cohort studies with adjustment for known confounding factors are required, especially in the current situation, where safe and effective COVID-19 vaccines are approved for pregnant and nursing mothers.

\section{Conclusion}

During the peak of the COVID-19 pandemic, the prevalence of breastfeeding initiation and EBF in the first six months postpartum were low among Saudi mothers in Riyadh. In our setting, we have Implemented policy that involve temporary maternal-neonatal separation in case of suspicions of or confirmed maternal COVID-19 test. The likelihood of EBF in early postpartum period was two to four times more among mothers who breastfed during their hospital stay, along with early initiation of breastfeeding. With new and evolving information regarding the current pandemic, infection control policies should support early and safe breastfeeding practices. Taking into consideration, the timely and efficient application of COVID-19 testing for mothers admitted for delivery.

\section{Abbreviations}

EBF, exclusive breastfeeding; EIBF, early initiation of breastfeeding; WHO, World Health Organization; UNICEF, United Nations International Children's Emergency Fund; UNFPA, United Nations Population Fund; ABM, Academy of Breastfeeding Medicine; RedCap, research electronic data capture; PCR, polymerase chain reaction; NSVD, normal spontaneous vaginal delivery.

\section{Data Sharing Statement}

All data used in the study are available to interested researchers upon request from the corresponding author 
after approval from the Institutional Review Board at PNU (contactirb@pnu.edu.sa).

\section{Ethics Approval and Informed Consent}

The study was conducted according to the guidelines of the Declaration of Helsinki and was approved by the Institutional Review Board at Princess Nourah bint Abdulrahman University (IRB:20-0419), which deemed the study exempt from further approval, due to the study posing no more than minimal risk to patients. All participants gave informed verbal consent before completing data collection tool, which was approved by the IRB.

\section{Acknowledgments}

I would like to express my thanks and gratitude to Princess Nourah bint Abdulrahman University Researchers Supporting Project number (PNURSP2022R289), Princess Nourah bint Abdulrahman University, Riyadh, Saudi Arabia, for funding this research. Special thanks to Ms Munirah Almalki, Ms Suhailah Al Shahrani, and Ms Sarah Almeshal for their substantial efforts in data collection. Also, I would like to thank Health Sciences Research Center at Princess Nourah bint Abdulrahman University for their support in revising data analysis. My appreciations to the cooperation of research office, infection prevention and control, and OB/GYN departments at King Abdullah bin Abdulaziz University Hospital (KAAUH), Princess Nourah bint Abdulrahman University.

\section{Funding}

This research was funded by Princess Nourah bint Abdulrahman University Researchers Supporting Project number (PNURSP2022R289), Princess Nourah bint Abdulrahman University, Riyadh, Saudi Arabia.

\section{Disclosure}

The author reports no conflicts of interest in this work.

\section{References}

1. Sankar MJ, Sinha B, Chowdhury R, et al. Optimal breastfeeding practices and infant and child mortality: a systematic review and meta-analysis. Acta Paediatr. 2015;104(467):3-13. doi:10.1111/ apa.13147

2. Chowdhury R, Sinha B, Sankar MJ, et al. Breastfeeding and maternal health outcomes: a systematic review and meta-analysis. Acta Paediatr. 2015;104:96-113. doi:10.1111/apa.13102
3. Kramer MS, Kakuma R. Optimal duration of exclusive breastfeeding. Cochrane Database Syst Rev. 2012;2012(8):CD003517. doi:10.1002/ 14651858.CD003517.pub2

4. Karimi FZ, Sadeghi R, Maleki-Saghooni N, Khadivzadeh T. The effect of mother-infant skin to skin contact on success and duration of first breastfeeding: a systematic review and meta-analysis. Taiwan J Obstet Gynecol. 2019;58(1):1-9. doi:10.1016/j.tjog.2018.11.002

5. World Health Organization. Health Topics: Breastfeeding. Geneva: WHO; 2012.

6. World Health Organization. Breastfeeding and COVID-19; 2020. Available from: https:/www.who.int/news-room/commentaries/ detail/breastfeeding-and-covid-19. Accessed September 10, 2021.

7. Gribble K, Marinelli KA, Tomori C, Gross MS. Implications of the COVID-19 pandemic response for breastfeeding, maternal caregiving capacity and infant mental health. J Hum Lact. 2020;36(4):591-603. doi: $10.1177 / 0890334420949514$

8. Brown A, Shenker N. Experiences of breastfeeding during COVID-19: lessons for future practical and emotional support. Matern Child Nutr. 2021;17(1):e13088. doi:10.1111/mcn.13088

9. Chambers C, Krogstad P, Bertrand K, et al. Evaluation for SARS-CoV-2 in breast milk from 18 infected women. JAMA. 2020;324(13):1347-1348. doi:10.1001/jama.2020.15580

10. El-Gilany A. COVID-19 and breastfeeding. Asp Biomed Clin Case Rep. 2020;3(2):102-105. doi:10.36502/2020/ASJBCCR.6194

11. World Health Organization. Breastfeeding advice during the COVID-19 outbreak; 2021. Available from: http://www.emro.who. int/nutrition/nutrition-infocus/breastfeedingadvice-during-covid-19outbreak.html. Accessed September 11, 2021.

12. Barrero-Castillero A, Beam KS, Bernardini LB, et al. COVID-19: neonatal-perinatal perspectives. J Perinatol. 2020;41(5):940-951. doi:10.1038/s41372-020-00874-x

13. Centers for Disease Control and Prevention. Interim considerations for infection prevention and control of coronavirus disease 2019 (COVID-19) in inpatient obstetric healthcare settings. Available form: https://www.cdc.gov/coronavirus/2019-ncov/hcp/inpatientobstetric-healthcare-guidance.html. Accessed September 11, 2021.

14. Wang L, Shi Y, Xiao T, et al. Chinese expert consensus on the perinatal and neonatal management for the prevention and control of the 2019 novel coronavirus infection (First edition). Ann Transl Med. 2020;8(3):47. doi:10.21037/atm.2020.02.2032154287

15. Moro GE, Bertino E. Breastfeeding, human milk collection and containers, and human milk banking: hot topics during the COVID-19 pandemic. J Human Lactation. 2020;36(4):604-608. doi:10.1177/0890334420934391

16. Stuebe A. Should infants be separated from mothers with COVID-19? First, do no harm. Breastfeed Med. 2020;15 (5):351-352. doi:10.1089/bfm.2020.29153.ams

17. Latorre G, Martinelli D, Guida P, et al. Impact of COVID-19 pandemic lockdown on exclusive breastfeeding in non-infected mothers. Int Breastfeed J. 2021;16(1):36. doi:10.1186/s13006-02100382-4

18. Pacheco F, Sobral M, Guiomar R, De la Torre-luque A, CaparrosGonzalez RA, Ganho-ávila A. Breastfeeding during COVID-19: a narrative review of the psychological impact on mothers. Behav Sci (Basel). 2021;11(3):34. doi:10.3390/bs11030034

19. Ahmed AE, Salih OA. Determinants of the early initiation of breastfeeding in the Kingdom of Saudi Arabia. Int Breastfeed $J$. 2019;14:13. doi:10.1186/s13006-019-0207-z

20. Alzaheb RA. Factors associated with the early introduction of complementary feeding in Saudi Arabia. Int J Environ Res Public Health. 2016;13:702. doi:10.3390/ijerph13070702

21. Raheel H, Tharkar S. Why mothers are not exclusively breast feeding their babies till 6 months of age? Knowledge and practices data from two large cities of the Kingdom of Saudi Arabia. Sudan J Paediatr. 2018;18:28-38. 
22. Al Shahrani AS, Hushan HM, Binjamaan NK, Binhuwaimel WA, Alotaibi JJ, Alrasheed LA. Factors associated with early cessation of exclusive breast feeding among Saudi mothers: a prospective observational study. J Family Med Prim Care. 2021;10:3657-3663. doi:10.4103/jfmpc.jfmpc_852_21

23. Al-Jassir M, Moizuddin SK, Al-Bashir B. A review of some statistics on breastfeeding in Saudi Arabia. Nutr Health. 2003;17:123-130. doi:10.1177/026010600301700203

24. Martin JA, Hamilton BE, Osterman MJ, Curtin SC, Matthews TJ. Births: final data for 2013. Natl Vital Stat Rep. 2015;64(1):1-65.

25. Noel-Weiss J, Boersma S, Kujawa-Myles S. Questioning current definitions for breastfeeding research. Int Breastfeed J. 2012;7(1):9. doi:10.1186/1746-4358-7-9

26. Exclusive breastfeeding under 6 months (\%). Available form: https:// www.who.int/data/gho/indicator-metadata-registry/imr-details/130. Accessed November 25, 2021.

27. Merewood A, Davanzo R, Haas-Kogan M, et al. Breastfeeding supportive practices in European hospitals during the COVID-19 pandemic. J Matern Fetal Neonatal Med. 2021;1-7. doi:10.1080/ 14767058.2021.1986482

28. Conti MG, Natale F, Stolfi I, et al. Consequences of early separation of maternal-newborn dyad in neonates born to SARS-CoV-2 positive mothers: an observational study. Int J Env Res Public Health. 2021;18(11):5899. doi:10.3390/ijerph18115899

29. Sakalidis VS, Rea A, Perrella SL, et al. Wellbeing of breastfeeding women in Australia and New Zealand during the COVID-19 pandemic: a cross-sectional study. Nutrients. 2021;13(6):1831. doi:10.3390/ nu13061831

30. Infant and young child feeding; 2021. Available from https://data. unicef.org/topic/nutrition/infant-and-young-child-feeding/. Accessed December 15, 2021.

31. Suresh S, Sharma K, Saksena M, Thukral A, Agarwal R, Suresh S. Vatsa, MPredictors of breastfeeding problems in the first postnatal week and its effect on exclusive breastfeeding rate at six months: experience in a tertiary care centre in northern India. Indian J Public Health. 2014;58(4):270-273. doi:10.4103/0019-557X.146292

32. Kozhimannil KB, Jou J, Attanasio LB, Joarnt LK, McGovern P, Laine K. Medically complex pregnancies and early breastfeeding behaviors: a retrospective analysis. PLoS One. 2014;9(8):e104820. doi:10.1371/journal.pone. 0104820

33. Feenstra MM, Jørgine Kirkeby M, Thygesen M, Danbjørg DB, Kronborg H. Early breastfeeding problems: a mixed method study of mothers' experiences. Sex Reprod Healthc. 2018;16:167-174. doi:10.1016/j.srhc.2018.04.003
34. Zitkute V, Snieckuviene V, Zakareviciene J, Pestenyte A, Jakaite V, Ramasauskaite D. Reasons for breastfeeding cessation in the first year after childbirth in Lithuania: a prospective cohort study. Medicina (Kaunas). 2020;56(5):226. doi:10.3390/medicina56050226

35. Bhatt N. Breastfeeding in India is disrupted as mothers and babies are separated in the pandemic. BMJ. 2020;370:m3316. doi:10.1136/bmj. $\mathrm{m} 3316$

36. Kotlyar AM, Grechukhina O, Chen A, et al. Vertical transmission of coronavirus disease 2019: a systematic review and meta-analysis. $\mathrm{Am}$ J Obstet Gynecol. 2021;224(1):35-53.e3. doi:10.1016/j.ajog.2020.07.049

37. Van Oosterhout C, Hall N, Ly H, Tyler KM. COVID-19 evolution during the pandemic - Implications of new SARS-CoV-2 variants on disease control and public health policies. Virulence. 2021;12 (1):507-508. doi:10.1080/21505594.2021.1877066

38. Arab News. Saudi Arabia ready to launch vaccine campaign. Available form: https://www.arabnews.com/node/1775896/saudi422arabia. Accessed September 23, 2021.

39. Alyami MH, Naser AY, Orabi MAA, Alwafi H, Alyami HS. Epidemiology of COVID-19 in the Kingdom of Saudi Arabia: an ecological study. Front Public Health. 2020;8:506. doi:10.3389/ fpubh.2020.00506

40. Al-Matary A, Almatari F, Al-Matary M, et al. Clinical outcomes of maternal and neonate with COVID-19 infection - multicenter study in Saudi Arabia. J Infect Public Health. 2021;14(6):702-708. doi:10.1016/j.jiph.2021.03.013

41. Hackman NM, Schaefer EW, Beiler JS, Rose CM, Paul IM. Breastfeeding outcome comparison by parity. Breastfeed Med. 2015;10(3):156-162. doi:10.1089/bfm.2014.0119

42. Colombo L, Crippa BL, Consonni D, et al. Breastfeeding determinants in healthy term newborns. Nutrients. 2018;10:48. doi:10.3390/ nu10010048

43. Gianni ML, Bettinelli ME, Manfra P, et al. Breastfeeding difficulties and risk for early breastfeeding cessation. Nutrients. 2019;11 (10):2266. doi:10.3390/nu11102266

44. Chang PC, Li SF, Yang HY, et al. Factors associated with cessation of exclusive breastfeeding at 1 and 2 months postpartum in Taiwan. Int Breastfeed J. 2019;14:18. doi:10.1186/s13006-019-0213-1
Risk Management and Healthcare Policy

\section{Publish your work in this journal}

Risk Management and Healthcare Policy is an international, peerreviewed, open access journal focusing on all aspects of public health, policy, and preventative measures to promote good health and improve morbidity and mortality in the population. The journal welcomes submitted papers covering original research, basic science, clinical \& epidemiological studies, reviews and evaluations, guidelines, expert opinion and commentary, case reports and extended reports. The manuscript management system is completely online and includes a very quick and fair peer-review system, which is all easy to use. Visit http://www.dovepress.com/testimonials.php to read real quotes from published authors. 